\title{
A salmon peptide diet alleviates experimental colitis as compared with fish oil
}

\author{
Tore Grimstad ${ }^{1,2}$, Bodil Bjørndal ${ }^{3}$, Daniel Cacabelos ${ }^{4}$, Ole G. Aasprong ${ }^{5}$, Roald Omdal ${ }^{2,6}$, \\ Asbjørn Svardal ${ }^{2}$, Pavol Bohov ${ }^{3}$, Reinald Pamplona ${ }^{4}$, Manuel Portero-Otin ${ }^{4}$, Rolf K. Berge ${ }^{3,7}$ and \\ Trygve Hausken ${ }^{2,8}$ \\ ${ }^{1}$ Division of Gastroenterology, Department of Medicine, Stavanger University Hospital, Stavanger, Norway \\ ${ }^{2}$ Institute of Medicine, University of Bergen, Bergen, Norway \\ ${ }^{3}$ Section of Clinical Biochemistry, Haukeland University Hospital, Bergen, Norway \\ ${ }^{4}$ Department of Experimental Medicine, University of Lleida (IRB Lleida), Lleida, Spain \\ ${ }^{5}$ Department of Pathology, Stavanger University Hospital, Stavanger, Norway \\ ${ }^{6}$ Department of Medicine, Stavanger University Hospital, Stavanger, Norway \\ ${ }^{7}$ Department of Heart Diseases, Haukeland University Hospital, Bergen, Norway \\ ${ }^{8}$ Division of Gastroenterology, Department of Medicine, Haukeland University Hospital, Bergen, Norway
}

(Received 22 May 2012 - Final revision received 31 August 2012 - Accepted 16 October 2012)

Journal of Nutritional Science (2013), vol. 2, e2, page 1 of 8

doi:10.1017/jns.2012.23

\section{Abstract}

Fish oil (FO) has been shown to have anti-inflammatory properties in animal models of inflammatory bowel disease, but how fish peptides (FP) influence intestinal inflammation has been less studied. Male Wistar rats, divided into five groups, were included in a 4-week dietary intervention study. Of the groups, four were exposed in the fourth week to $5 \%$ dextran sulfate sodium (DSS) to induce colitis, while one group was unexposed. The diets were: (1) control, (2) control + DSS, (3) FO (5\%) + DSS, (4) FP (3.5\%) + DSS, (5) FO + FP + DSS. Following DSS intake, weight and disease activity index (DAI) were assessed, and histological combined score (HCS), selected colonic PG, cytokines, oxidative damage markers and mRNA levels were measured. FP reduced HCS, tended to lower DAI $(P=0.07)$ and reduced keratinocyte chemoattractant/growth-regulated oncogene levels, as compared with the FO diet. FP also reduced mRNA levels of Il-6 and Cxcl1, although not significantly. FO intake increased the DAI as compared with DSS alone. $\mathrm{PGE}_{3}$ levels increased after the FO diet, and even more following FO + FP intake. The FP diet seems to have a protective effect in DSS-induced colitis as compared with FO. A number of beneficial, but non-significant, changes also occurred after FP $v$. DSS. A combined FO + FP diet may influence PG synthesis, as $\mathrm{PGE}_{3}$ levels were higher after the combined diet than after $\mathrm{FO}$ alone.

Key words: Colitis: Diet therapy: Inflammatory bowel diseases: $n$-3 Fatty acids

Ulcerative colitis (UC) is an inflammatory bowel disease (IBD) with a chronic relapsing course, affecting the colon mucosa to a variable extent and resulting in bloody diarrhoea ${ }^{(1)}$. Dextran sulfate sodium (DSS)-induced colitis has been used as an experimental model for UC, being clinically and histomorphologically reminiscent of the human disease. Dietary $n$ - 3 PUFA in experimental rodent models of IBD, including DSS colitis, have shown beneficial effects ${ }^{(2,3)}$. However, data concerning the anti-inflammatory effect of marine PUFA supplements in patients with $\mathrm{UC}$ are inconsistent ${ }^{(4-6)}$. It is therefore of interest

Abbreviations: DAI, disease activity index; DSS, dextran sulfate sodium; FO, fish oil; FP, fish peptide; GRO, growth-regulated oncogene; HCS, histological combined score; $\mathrm{IBD}$, inflammatory bowel disease; $\mathrm{KC}$, keratinocyte chemoattractant; UC, ulcerative colitis.

* Corresponding author: Tore Grimstad, fax +47 51519923, email grto@sus.no 
to investigate whether dietary supplements other than fish oil (FO) can have beneficial effects on intestinal inflammation. FO may have anti-inflammatory effects through several mechanisms, including an alteration of the substrate from $20: 4 n-6$ (arachidonic acid) to $20: 5 n-3$ (EPA) and $22: 6 n-3$ (DHA) in cell membrane phospholipids. This results in eicosanoids that have lower inflammatory potential, such as $\mathrm{PGE}_{3}$ and leukotriene $\mathrm{B}_{5}{ }^{(7)}$. Intake of $\mathrm{FO}$ reduced mononuclear cell production of pro-inflammatory cytokines such as IL-1 $\beta$ and TNF $\alpha$ after endotoxin stimulation ${ }^{(8)}$.

Fish peptides (FP) seem to have tissue reparative properties based on several small studies in animals and human subjects. A commercially available fish hydrolysate, containing 75-80 $\%$ protein constituents, reduced non-steroidal anti-inflammatory drug (NSAID)-induced mucosal injury in small intestine in mice ${ }^{(9)}$. NSAID-induced damage in small intestine and dyspeptic symptoms decreased in human volunteers ${ }^{(10)}$. Furthermore, a dietary supplement containing bioactive peptides and amino acids seemed to beneficially affect symptoms and intestinal permeability in IBD patients ${ }^{(11)}$.

In a previous study, we found that regular intake of Atlantic salmon for 8 weeks in mildly active UC patients reduced the symptoms of disease activity and improved the fatty acid profile in plasma as well as in colon samples ${ }^{(12)}$. Fish fillets may contain bioactive proteins and peptides in addition to marine fatty acids. Oxidative stress, caused by an imbalance between the formation of reactive oxygen species and counteracting antioxidants occurs in several chronic inflammatory conditions, including $\mathrm{IBD}^{(13)}$. Increasing the antioxidant levels might reduce tissue damage and the inflammatory process. Fish and fish protein may have such an antioxidant potential. A peptide fraction from fish hydrolysate seemed to have an antioxidant effect compared with the natural antioxidant $\alpha$-tocopherol ${ }^{(14)}$. In obese subjects consuming cod, the concentration of malondialdehyde, a measure of lipid oxidation, was reduced in blood $^{(15)}$.

The aim of this study was to evaluate possible antiinflammatory effects of dietary hydrolysed salmon peptides, also named fish peptides (FP), in DSS-induced colitis in rats. Furthermore, our aim was to compare dietary intervention with FP to that of FO or combined FO + FP and assess the impact on disease activity, selected markers of inflammation and protein oxidative damage in colon tissue samples.

\section{Experimental methods}

\section{Animals and husbandry}

A total of fifty male Wistar rats (Taconic), 12 weeks old, with a weight of approximately $360 \mathrm{~g}$ at arrival, were placed in Makrolon III cages in an open system, with five animals in each cage. They were kept under standard laboratory conditions with $12 \mathrm{~h}$ dark-12 h light cycles and temperature $22 \pm$ $1^{\circ} \mathrm{C}$, relative humidity $55 \pm 5 \%$ and twenty air changes per h. The rats had free access to food and tap water. The experiment was conducted according to the Guidelines for Care and Use of Experimental Animals, and the protocol was approved by the Norwegian State Board of Biological Experiments with Living Animals.

\section{Induction of colitis}

DSS (MW 44000; TdB Consultancy AB) at $50 \mathrm{~g} / 1$ was administered in drinking water as previously described ${ }^{(16)}$ for $7 \mathrm{~d}$ to induce colitis.

\section{Experimental protocol}

The rats were acclimatised for $7 \mathrm{~d}$ and then separated into five groups of ten rats. The following diets were given: (1) control, (2) DSS + control, (3) DSS + FO (5\%), (4) DSS + FP (3.5\%) and (5) DSS + FO + FP. All groups had free access to food on days 1-29, and DSS was administered during the last week of the experiment in groups 2-5. All rats were killed the day after DSS treatment was discontinued - on day 30. At that time the animals were anaesthetised by inhalation of Isoflurane ( $2 \%$; 'Isoba vet', Intervet/Schering-Plough Animal Health) in an anaesthesia chamber. Thoracotomy, cardiac puncture and exsanguination were then performed.

\section{Diet composition}

The control diet consisted of a standard casein diet with $20 \%$ (w/w) protein (Sigma-Aldrich), and $7 \%$ fat, composed of $5 \%$ lard (a generous gift from Ten Kate Vetten BV) and $2 \%$ soyabean oil (Dyets Inc.). Lard was replaced by $5 \%$ FO in group 3 (EPAX 4020 TG, EPAX AS), and casein partly by $3.5 \%$ FP hydrolysate (Marine BioProducts AS) in group 4, and both in group 5. The FP were produced from salmon by-products (spine) by enzymatic hydrolysis using controlled autolysis with commercial proteases. The resulting hydrolysate was further fractionated using different forms of filtration including ultrafiltration. The sizes of the peptides were $<10000 \mathrm{Da}$, with $70 \%$ of total peptides $<1000 \mathrm{Da}$. The final product consisted of $>90 \%$ peptides. The other constituents of the diets were maize starch, dyetrose, sucrose, fibre, American Institute of Nutrition (AIN)-93G mineral mix, AIN-93 vitamin mix,

Table 1. Composition of the experimental diets ( $\mathrm{g} / \mathrm{kg}$ diet)*

\begin{tabular}{lcccc}
\hline Ingredients & Control & FO & FP & FO + FP \\
\hline FO & & 50 & & 50 \\
Lard & 50 & & 50 & \\
Soyabean oil & 20 & 20 & 20 & 20 \\
FP & & & 35 & 35 \\
Casein & 200 & 200 & 165 & 165 \\
Maize starch & 397 & 397 & 397 & 397 \\
Dyetrose & 132 & 132 & 132 & 132 \\
Sucrose & 100 & 100 & 100 & 100 \\
Fibre & 50 & 50 & 50 & 50 \\
AIN-93G mineral mix & 35 & 35 & 35 & 35 \\
AIN-93 vitamin mix & 10 & 10 & 10 & 10 \\
L-Cysteine & 3 & 3 & 3 & 3 \\
Choline bitartrate & 2.5 & 2.5 & 2.5 & 2.5 \\
tert-Butyl-hydroquinone & 0.014 & 0.014 & 0.014 & 0.014 \\
\hline
\end{tabular}

FO, fish oil; FP, fish peptides; AIN, American Institute of Nutrition.

* The diets were isonitrogenous and isoenergetic. 
L-cysteine, choline bitartrate (Dyets Inc.), and tert-butylhydroquinone (Sigma-Aldrich) (Table 1).

\section{Weight, food intake and disease activity}

Body weight and food intake were measured before DSS administration (day 23) and daily during DSS intake days 24-29. The disease activity index (DAI) is a composite score where weight loss, stool consistency and rectal bleeding are rated from 0 to 4 , and the total sum is divided by $3^{(17)}$. DAI was assessed on the last day of colitis induction (day 30 ).

\section{Fatty acid composition of the diets}

The fatty acids were measured as previously described ${ }^{(17)}$.

\section{Sample collection}

After killing, the major part of the colon, from the colocaecal junction to the anal verge was removed, and the length was measured unstretched. The colon was rinsed in PBS, and samples from both the ends and the middle part were separated, then divided longitudinally and placed in $10 \%$ paraformaldehyde. The samples were later embedded in paraffin and sectioned before staining with haematoxylin and eosin for histopathological examination. The remaining colon was divided into one distal and proximal segment. Pieces from both segments were cooled in liquid $\mathrm{N}_{2}$, freeze clamped and then stored at $-80^{\circ} \mathrm{C}$ for further analyses.

\section{Further analyses}

Colon specimen. In the haematoxylin and eosin-stained tissue samples, the crypt and inflammatory scores were determined according to a validated scoring system $^{(18)}$ and examined by a pathologist unaware of the experimental protocol. Crypt injury and inflammation severity scores were assessed as described earlier ${ }^{(17)}$. Both the crypt and inflammatory score include a measure of involvement: grade $1,1-25 \%$; grade $2,26-50 \%$; grade 3, 51-75\%; grade 4 , $76-100 \%$. The score is the product of either the crypt grade by involvement grade or inflammation grade by the involvement grade. The histological combined score (HCS) was the sum of the crypt and inflammation scores, and was measured in all three locations of the colon.

Preparations for tissue cytokine measurements. Full wall sections of the colon were used, and $50 \mathrm{mg}$ samples were homogenised and prepared as described previously ${ }^{(17)}$.

Tissue cytokine analyses. Custom-made rat cytokine fourplex kits including IL-1 $\beta$, IL-5, interferon- $\gamma$ and keratinocyte chemoattractant (KC)/growth-regulated oncogene (GRO) and single-plex kits with TNF $\alpha$ (Meso Scale Discovery) were used to measure cytokines in the supernatant from the homogenisate. The analyses were performed according to the manufacturer's recommendations. Results are given as $\mu \mathrm{g} / \mathrm{kg}$ (wet tissue).

PG. Chemicals, solutions and sample preparation of frozen colon tissue samples (12-35 mg) were performed as described ${ }^{(17)}$.

LC/MS/MS. The LC system was an Agilent 1200 Series with a binary pump, a variable volume injector and a thermostated autosampler. HPLC separation was conducted as described ${ }^{(17)}$. Multiple reaction monitoring for data acquisition and negative ion detection was used. MassHunter software (Agilent) was used for HPLC system control, data acquisition and data processing. The PG analysed are expressed as $\mu \mathrm{g} / \mathrm{kg}$ (wet tissue).

Markers of oxidative stress. Glutamic semialdehyde is a carbonyl product that results from direct oxidative damage of amino acids (arginyl and prolyl residues). Carboxyethyllysine is derived from glycosylation reactions on proteins and carboxymethyl-lysine from both glycoxidation and lipoxidation reactions. Glutamic semialdehyde, carboxymethyllysine and carboxyethyl-lysine concentrations were determined as trifluoroacetic acid methyl esters derivates in acidhydrolysed, delipidated and reduced intestine protein samples. Homogenisates were measured by GC/MS as previously described ${ }^{(19)}$. Protein samples were prepared, and internal standards were added as described ${ }^{(17,19)}$. GC/MS analyses were carried out as previously described ${ }^{(17)}$. Quantification was performed by external standardisation using standard curves constructed from mixtures of ${ }^{2} \mathrm{H}$-labelled and non- ${ }^{2} \mathrm{H}$-labelled standards. Analytes were detected by selected ion-monitoring GC/MS ${ }^{(17)}$. The amounts of products were expressed as the ratio of micromol per mol lysine.

Gene expression analysis. Total cellular RNA was purified from $20 \mathrm{mg}$ frozen tissue samples from distal colon, RNA was quantified spectrophotometrically, and the quality was evaluated as described ${ }^{(17)}$. For each sample, $4 \mu \mathrm{g}$ total RNA was reversely transcribed in $100 \mu \mathrm{l}$ reactions as previously reported ${ }^{(17)}$. Real-time PCR was performed with custom-made 384-well microfluidic plates (Taq-Man Low Density Arrays; Applied Biosystems) on the following genes: Il-1b, Rn00580432_m1; Il-6, Rn 00561420; KC-GRO (KC/ GRO, Cxcl1), Rn00578225_m1; inducible nitric oxide synthase 2 (Nos2), Rn00561646_m1; PPAR $\gamma$ (Pparg), Rn00440945; PPAR $\gamma$ cofactor 1a (Ppargc-1a), Rn00580241; Sirtuin 1 (Sirt1), Rn01428093_m1; PGD 2 synthase (Ptgds), Rn00564605_m1; PGE synthase (Ptges), Rn00572047_m1; PG-endoperoxide synthase 2 (Cox-2, Ptgs2), Rn01483828_m1.

All probes and primers were obtained from Applied Biosystems. Two different control genes were included: $18 s$ (Kit-FAM-TAMRA (reference RT-CKFT-18s)) from Eurogentec; and Rplp0 (Rn00821065_g1) from Applied Biosystems. To normalise the absolute quantification according to the reference genes, a second set of PCR was performed for all experimental samples, and the relative abundance values were calculated for the reference genes as well as for the target 
genes using standard curves derived from Universal Rat Reference RNA (Agilent Technologies Inc.). NormFinder ${ }^{(20)}$ software was used to evaluate the reference genes, and the relative abundance value of each gene in each sample was normalised against the value of the most stable reference gene, Rplp0.

\section{Statistics}

The Shapiro-Wilk test was used to test for normality of data. The Student's $t$ test or one-way ANOVA was used to analyse differences between two or more groups, respectively. The Mann-Whitney rank test or the Kruskal-Wallis test was applied in not normally distributed data for two or more groups. Post hoc tests were performed by the Mann-Whitney rank test. A total of four pre-determined pairwise differences were analysed: FP $v$. DSS, FO $v$. DSS, FP $v$. FO and FO $v$. FO + FP. Bonferroni corrections were made in the case of multiple group analyses.

For all tests a $P$ value $\leq 0 \cdot 05$ was considered as statistically significant. SPSS 15.0 and PASW Statistics 18 statistical software packages were used for analyses (SPSS Inc.). Bonferroni corrections were not performed in the gene expression analyses.

\section{Results}

\section{Fatty acid composition in the diets}

As intended, the FO and FO + FP diets contained large relative amounts of EPA and DHA, as well as a high total level of n-3 PUFA. The FP diet had low levels of n-3 PUFA, similar to the control diet. In the FO-containing diets, we observed a relative decrease in levels of $18: 1 n-9$ as the $n$-3 PUFA levels increased (Table 2).

\section{Weight gain, feed intake and dextran sulfate sodium intake}

During DSS intake all animal groups lost weight significantly compared with the control group, which in contrast gained weight. Feed intake was similar in the DSS-exposed groups, while the controls had higher food consumption (Table 3). Of the animals in the FO + FP group, two died during the DSS week.

\section{Histological combined score}

When dividing all DSS-exposed rats into groups according to the proximal, middle or distal colon segment, there was a significant difference in HCS between the three segments $(P=0.001)$. Post hoc analyses showed a significantly higher HCS in the distal and middle colon segments $v$. the proximal segment $(P=0.002$ for both differences). In the distal colon, the DSS group had a significantly higher HCS than controls ${ }^{(17)}$. There was an almost significant difference between DSS-exposed groups $(P=0 \cdot 07)$. Post hoc analyses showed a reduced HCS after the FP diet (median 13.5; range 8-21) $v$. the FO diet (median 19; range 15-28) $(P=0 \cdot 03$ ). Neither FO nor FP reduced HCS $v$. DSS (median 17.5; range $1-20$ ) ( $P=1.0$ for both differences). HCS did not differ significantly between $\mathrm{FO}$ and $\mathrm{FO}+\mathrm{FP}$ (median 15; range 4-21) $(P=0 \cdot 08)$ (Table 4). Histology sections illustrating the median HCS from all study groups are shown in Fig. 1.

\section{Disease activity index}

The median DAI value in the DSS group was higher than in the control group ${ }^{(17)}$. DAI differed significantly between DSS-exposed groups $(P=0 \cdot 02)$. Post hoc tests showed no significant reduction of DAI in the FP group (median 1.3; range $0.7-3.7) v$. the $\mathrm{FO}$ group (median $2 \cdot 8$; range $1.3-3 \cdot 7$ ) $(P=0 \cdot 07)$. DAI was higher after the FO diet than after DSS alone (median 1.0; range $0.3-3 \cdot 0)(P=0.04)$. DAI did not differ between FP and DSS $(P=1 \cdot 0)$, and did not differ in the $\mathrm{FO} v$, the $\mathrm{FO}+\mathrm{FP}$ group (median 1.3 range $0.7-$ 3.7) $(P=0 \cdot 6)$ (Table 4).

Table 2. Fatty acid compositions of the diets*

\begin{tabular}{|c|c|c|c|c|}
\hline & Control & $\mathrm{FO}$ & FP & $\mathrm{FO}+\mathrm{FP}$ \\
\hline $14: 0$ & 1.29 & 0.69 & 1.31 & 0.65 \\
\hline $16: 0$ & $20 \cdot 76$ & 5.55 & 21.83 & 5.52 \\
\hline $16: 1 n-7$ & 1.21 & 1.05 & 1.28 & 1.08 \\
\hline $18: 0$ & $12 \cdot 30$ & $3 \cdot 31$ & $13 \cdot 10$ & 3.31 \\
\hline $18: 1 n-9$ & 29.53 & $10 \cdot 49$ & $31 \cdot 21$ & $10 \cdot 58$ \\
\hline $18: 1 n-7$ & 1.93 & $2 \cdot 18$ & 2.05 & $2 \cdot 23$ \\
\hline $18: 2 n-6$ & $22 \cdot 10$ & 14.99 & 23.23 & $15 \cdot 22$ \\
\hline $18: 3 n-3$ & 2.57 & $2 \cdot 24$ & $2 \cdot 70$ & $2 \cdot 27$ \\
\hline $18: 4 n-3$ & 0.003 & 1.28 & 0.006 & 1.32 \\
\hline $20: 4 n-6$ & 0.14 & 1.31 & 0.15 & 1.33 \\
\hline $20: 5 n-3$ & 0.02 & $25 \cdot 63$ & 0.07 & $26 \cdot 08$ \\
\hline $22: 5 n-3$ & 0.01 & 3.50 & 0.03 & 3.50 \\
\hline $22: 6 n-3$ & 0.03 & $19 \cdot 24$ & 0.07 & $19 \cdot 35$ \\
\hline SFA & 35.64 & 10.85 & 37.53 & $10 \cdot 71$ \\
\hline MUFA & 33.60 & $16 \cdot 21$ & 35.52 & $16 \cdot 37$ \\
\hline PUFA ( $n-3)$ & $2 \cdot 74$ & 54.31 & 2.98 & 54.98 \\
\hline PUFA $(n-6)$ & $22 \cdot 69$ & $18 \cdot 48$ & 23.86 & $17 \cdot 81$ \\
\hline PUFA $(n-3: n-6)$ & 0.12 & 2.94 & 0.12 & 3.09 \\
\hline $\mathrm{DBI}$ & 0.88 & 3.44 & 0.93 & 3.44 \\
\hline
\end{tabular}


Table 3. Average weight gain (\%) and feed intake per Wistar rat (g/d) during the dextran sodium sulfate (DSS) week $\dagger$ (Mean values with their standard errors for nine or ten animals per group)

\begin{tabular}{|c|c|c|c|c|c|}
\hline \multirow[b]{2}{*}{ Treatment } & \multicolumn{2}{|c|}{$\begin{array}{l}\text { Weight gain in DSS week } \\
\text { (\% per week) }\end{array}$} & \multicolumn{2}{|c|}{$\begin{array}{c}\text { Feed intake in DSS week } \\
\text { (g/d per rat) }\end{array}$} & \multirow{2}{*}{$\begin{array}{c}\text { DSS intake in DSS } \\
\text { week (ml/g rat) }\end{array}$} \\
\hline & Mean & SEM & Mean & SEM & \\
\hline Control & 4.62 & 1.48 & 21.55 & 0.75 & - \\
\hline Control + DSS & $-6.50^{\star}$ & $3 \cdot 16$ & $15 \cdot 70$ & 0.82 & 0.30 \\
\hline $\mathrm{FO}+\mathrm{DSS}$ & $-9 \cdot 88^{\star}$ & $2 \cdot 69$ & 14.54 & $1 \cdot 18$ & 0.36 \\
\hline$F P+D S S$ & $-6 \cdot 84^{\star}$ & $5 \cdot 34$ & $16 \cdot 17$ & 0.63 & 0.27 \\
\hline $\mathrm{FO}+\mathrm{FP}+\mathrm{DSS}$ & $-8 \cdot 90^{\star}$ & 5.00 & $16 \cdot 18$ & 0.86 & 0.47 \\
\hline
\end{tabular}

FO, fish oil; FP, fish peptides.

* Mean value was significantly different from that of the control group ( $P \leq 0.05$; one-way ANOVA, performed only on the weight results).

† Diets were FO, FP or a combination $(F O+F P)$ for 4 weeks, with DSS exposure in the last week of the experiment.

\section{Colon length}

The controls had a significantly longer colon than the DSS group, reflecting a colitis-induced shortening after DSS exposure $^{(17)}$. There were no differences in colon length between the DSS-exposed groups $(P=0 \cdot 20)$, but there was a tendency to have a longer colon in the FP group (data not shown).

$P G$

$\mathrm{PGE}_{3}$ levels were significantly different between the DSSexposed groups in the distal colon $(P=0 \cdot 001)$. Post hoc analyses showed that the FO diet increased $\mathrm{PGE}_{3}$ levels $v$. DSS alone. $\mathrm{PGE}_{3}$ levels were not significantly different in the FP $v$. the DSS group. The combined FO + FP group gave an additional increase of $\mathrm{PGE}_{3}$ as compared with the FO group (Table 5). In the case of $\mathrm{PGE}_{2}$, the difference between DSS groups was significant $(P=0.04)$ in the distal colon. However, none of the preselected post hoc tests revealed any differences. The largest difference in $\mathrm{PGE}_{2}$ levels occurred between the DSS and the $\mathrm{FO}+\mathrm{FP}$ group. For $\mathrm{PGD}_{2}$, a significant difference $(P=$ 0.04), was detected between DSS groups. In the distal colon, the $\mathrm{FO}+\mathrm{FP}$ diet increased $\mathrm{PGD}_{2}$ compared with $\mathrm{FO}$ (Table 5).

\section{Gene expression}

The expression of mRNA was significantly increased after DSS alone $v$. control for several selected genes, including $I l-6, I l-1 b$,
Cxcl1 and Ptges ${ }^{(17)}$. The FP diet reduced the mRNA levels of these genes compared with DSS alone, although not reaching significant levels. Nos2 expression was significantly different between the DSS-exposed groups $(P<0 \cdot 05)$. FP, FO and $\mathrm{FO}+\mathrm{FP}$ diets increased Nos2 mRNA levels $v$. DSS, although only significantly for the FO + FP diet (Table 5).

\section{Cytokines}

The protein levels of TNF $\alpha, \mathrm{IL}-1 \beta$ and $\mathrm{KC} / \mathrm{GRO}$ in the distal colon were in accord with mRNA levels. All three cytokines were significantly higher in the DSS animals $v$. the controls ${ }^{(17)}$. $\mathrm{KC} / \mathrm{GRO}$ was close to significantly different between the DSS-exposed groups $(P=0 \cdot 07)$. Post hoc tests revealed that the FP diet significantly lowered the KC/GRO levels $v$. the FO diet. The other preselected pairs analysed showed no significant differences. No significant differences in TNF $\alpha$ or IL- $\beta$ occurred between groups that received DSS.

\section{Markers of oxidative stress}

No significant differences were detected between the DSSexposed groups or between selected pairs for the three markers (data not shown). The levels were higher in the DSS $v$. the controls for all three markers although not reaching significance. We noted that, compared with DSS, median levels of all three markers tended to decrease after FO, while carboxyethyllysine and carboxymethyl-lysine levels were lower after FP, although not significantly.

Table 4. Histological combined scores (HCS) and disease activity index (DAI) in distal colon sections of Wistar rats fed fish oil (FO), fish peptides (FP) or a combination (FO + FP) for 4 weeks, and treated with dextran sodium sulfate (DSS) during the last week of the experiment (Median values and ranges)

\begin{tabular}{|c|c|c|c|c|c|c|c|c|}
\hline \multirow[b]{2}{*}{ Treatment } & \multicolumn{2}{|c|}{ HCS } & \multicolumn{2}{|c|}{ DAI } & \multicolumn{2}{|c|}{ HCS post hoc ${ }^{*}$} & \multicolumn{2}{|c|}{ DAI post hoc ${ }^{*}$} \\
\hline & Median & Range & Median & Range & Pair & $P$ & Pair & $P$ \\
\hline Control & 4.0 & $0-12$ & 0 & $0-0$ & & & & \\
\hline Control + DSS & 17.5 & $1-20$ & 1.0 & $0.3-3.0$ & FO $v$. DSS & 1.0 & FO $v$. DSS & 0.04 \\
\hline $\mathrm{FO}+\mathrm{DSS}$ & $19 \cdot 0$ & $15-28$ & $2 \cdot 8$ & $1 \cdot 3-3 \cdot 7$ & FO $v . F P$ & 0.03 & $\mathrm{FO} v . \mathrm{FP}$ & 0.07 \\
\hline$F P+D S S$ & 13.5 & $8-21$ & 1.3 & $0.7-3.7$ & FP $v$. DSS & 1.0 & FP $v$. DSS & 1.0 \\
\hline $\mathrm{FO}+\mathrm{FP}+\mathrm{DSS}$ & $15 \cdot 0$ & $4-21$ & 1.3 & $0.7-3.7$ & $\mathrm{FO} v . \mathrm{FO}+\mathrm{FP}$ & 0.08 & $\mathrm{FO} v \cdot \mathrm{FO}+\mathrm{FP}$ & 0.6 \\
\hline
\end{tabular}

* Post hoc tests analysed by the Mann-Whitney rank test. 


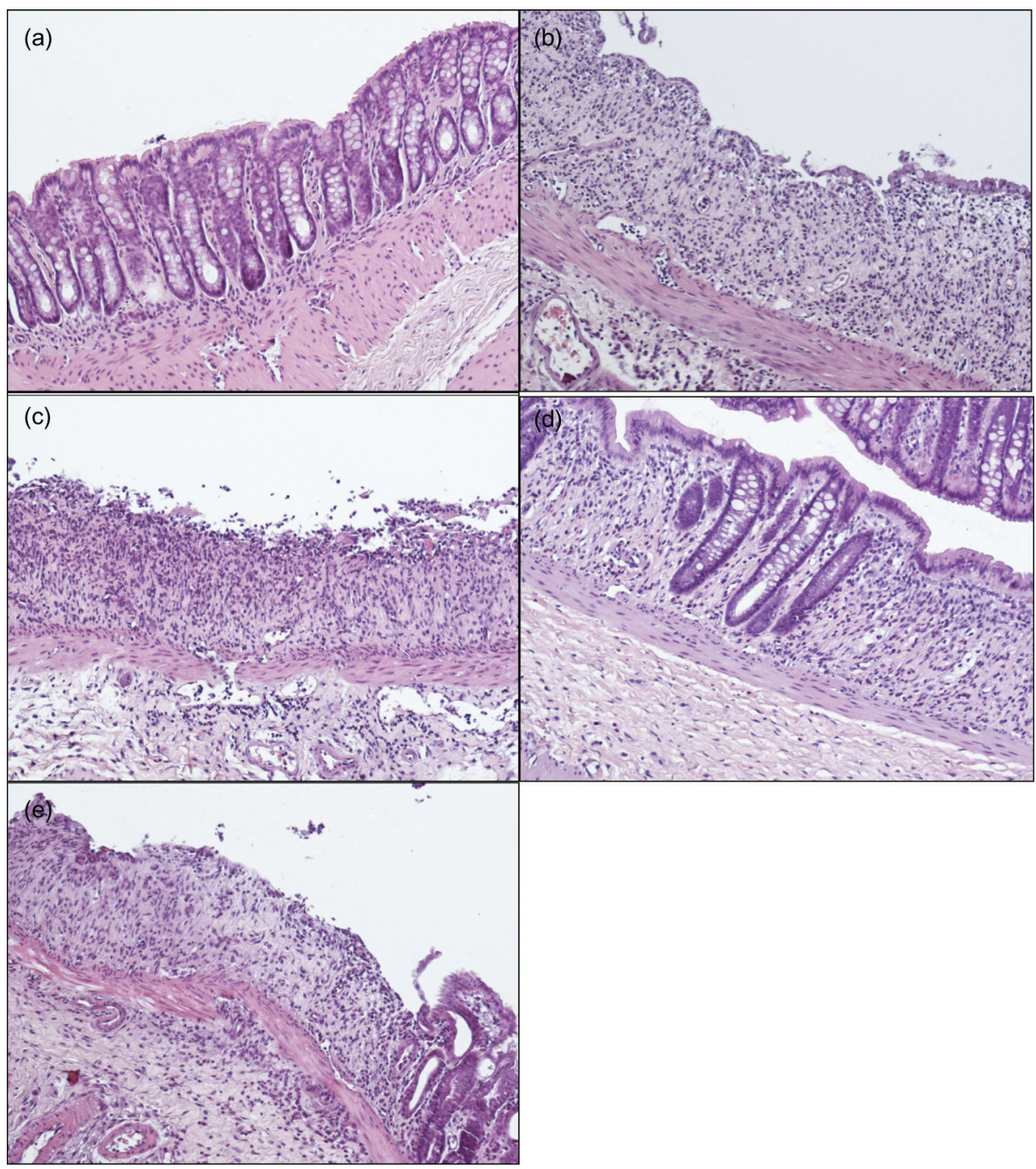

Fig. 1. Micrograph panels. (a) Normal colonic mucosa from a healthy control rat. (b) Colonic mucosa from a rat with dextran sulfate sodium (DSS) colitis showing crypt destruction and moderate inflammation, with almost intact epithelium. (c) Colonic mucosa from a rat after DSS and fish oil (FO) diet showing crypt destruction, severe inflammation and ulcerated surface. (d) Colonic mucosa from a rat after DSS and fish peptides (FP) diet showing some preserved normal crypts and some crypt destruction, moderate inflammation and intact epithelium. (e) Colonic mucosa from a rat after FO + FP diet showing destruction of most crypts, moderate inflammation and lost epithelium. Haematoxylin and eosin staining, magnification, $\times 400$.

Table 5. Selected gene expression, and cytokine and prostaglandin levels in distal colon sections of Wistar rats fed fish oil (FO), fish peptides (FP) or a combination (FO + FP) for 4 weeks, and treated with dextran sodium sulfate (DSS) during the last week of the experiment+ (Median values and ranges)

\begin{tabular}{|c|c|c|c|c|c|c|c|c|c|c|}
\hline & \multicolumn{2}{|c|}{ Control } & \multicolumn{2}{|c|}{ DSS } & \multicolumn{2}{|c|}{$\mathrm{DSS}+\mathrm{FO}$} & \multicolumn{2}{|c|}{$\mathrm{DSS}+\mathrm{FP}$} & \multicolumn{2}{|c|}{$\mathrm{DSS}+\mathrm{FO}+\mathrm{FP}$} \\
\hline & Median & Range & Median & Range & Median & Range & Median & Range & Median & Range \\
\hline \multicolumn{11}{|l|}{ Gene } \\
\hline Nos2 & 1.00 & $0.33-20.4$ & $58.5^{\star \star a}$ & $0.95-251$ & & & & & $272^{\mathrm{b}}$ & $99 \cdot 8-442$ \\
\hline \multicolumn{11}{|l|}{ Cytokine } \\
\hline KC/GRO & 0.70 & $0.38-1.12$ & $5.03^{* *}$ & $0.47-180$ & $12 \cdot 0^{\mathrm{a}}$ & $5 \cdot 07-124$ & $3 \cdot 20^{b}$ & $1 \cdot 13-64.6$ & & \\
\hline \multicolumn{11}{|c|}{ Prostaglandins } \\
\hline $\mathrm{PGE}_{3}$ & 0 & $0-0$ & $0^{\mathrm{a}}$ & $0-0.73$ & $13 \cdot 8^{\mathrm{b}}$ & $2.50-34.6$ & $1.53^{\mathrm{a}, \mathrm{c}}$ & $0-17 \cdot 2$ & $35.9^{c}$ & $10 \cdot 2-111$ \\
\hline $\mathrm{PGD}_{2}$ & $96 \cdot 2$ & $27 \cdot 5-507$ & $102^{\mathrm{a}}$ & $20 \cdot 8-272$ & $79 \cdot 3^{a}$ & $41 \cdot 4-156$ & & & $210^{b}$ & 51.9-343 \\
\hline
\end{tabular}

Nos2, inducible nitric oxide synthase 2; KC, keratinocyte chemoattractant; GRO, growth-regulated oncogene.

a,b,c Median values for the DSS groups with unlike superscript letters were significantly different $(P<0.05$ (Kruskal-Wallis test/Mann-Whitney post hoc test) except KC/GRO: $P=0.07$ (Kruskall-Wallis test)).

** Median value was significantly different from that of the control treatment $(P<0.01$; Mann-Whitney rank test).

† Nos2 expression values were normalised against an endogenous control, and variables are given as medians relative to control ( $n 7-8)$. Cytokine and prostaglandin levels ( $\mu$ g/kg wet tissue) are given as medians ( $n$ 8-10). 


\section{Discussion}

The main findings of this study are that hydrolysed salmon peptides, a novel dietary component used in IBD models, attenuated DSS-induced colitis as compared with FO. This was observed by fewer signs of histopathological inflammation, less clinical disease activity, a lowered inflammatory cytokine signature at the protein and mRNA levels, and increased $\mathrm{PGD}_{2}$. Also, the FP diet improved a number of variables when compared with animals fed DSS alone, such as cytokines and markers of protein oxidation, although not reaching statistical difference. In addition, we observed that the expected increase of $\mathrm{PGE}_{3}$ induced through metabolism of EPA from dietary $\mathrm{FO}$ increased further after combining $\mathrm{FO}$ and FP in the diet.

Although intake of FP has not been much investigated in experimental colitis, other dietary bioactive peptides have shown antiinflammatory effects in IBD animal models ${ }^{(21)}$. An intestinal protective effect from fish hydrolysate was suggested, as proliferation of HT 29 cells increased, while indomethacin-induced apoptose and small-intestinal villous damage were decreased in mice ${ }^{(9)}$. Moreover, intake of fish protein hydrolysate containing $75-80 \%$ protein constituents in healthy humans reduced small-intestinal damage caused by indomethacin ${ }^{(10)}$. The proposed mechanisms in these two studies were the presence of glutamine $(14-16 \%$ of the total), an antioxidant effect, and an effect of the $6-10 \%$ content of FO in the hydrolysate. The doses of $25-50 \mathrm{~g} / 1$ and $3 \mathrm{~g}$ daily used in their studies are not directly comparable with $35 \mathrm{~g} / \mathrm{kg}$ used in our study. FP contained $>90 \%$ peptides, but had a lower glutamine content of $8.5 \%(w / w)$. Glutamine, a substrate for enterocyte metabolism, could act protectively by preserving intestinal integrity and barrier function, as shown in previous experimental colitis models ${ }^{(22)}$. The $n$ - 3 content in FP was only $3.0 \%$, similar to the controls in our study $(2.7 \%)$, and can probably not explain the beneficial changes after the FP diet. Specific structural features have been linked to potentially bioactive peptides; the molecular weight should be $<10 \mathrm{kDa}$, most preferably $<1 \mathrm{kDa}$, and the size of peptides between two and twenty amino acids ${ }^{(23)}$. Our FP product is within this range.

DSS-induced experimental colitis has some similarities to the human disease UC. Epithelial injury is fundamental in this model, and during the acute colitis phase innate immune responses are activated ${ }^{(24)}$. The pro-inflammatory cytokine levels in the DSS model were at their lowest after the FP diet at both the protein and mRNA levels, suggesting an antiinflammatory effect from bioactive FP, influencing innate immunity. $\mathrm{PGD}_{2}$ reduces granulocyte infiltration in experimental colitis ${ }^{(25)}$, and its synthesis is up-regulated in UC patients in remission ${ }^{(26)}$. The tendency for $\mathrm{PGD}_{2}$ to increase after FP diets in our study may point to a protective effect.

Contrary to expectations, FO did not improve signs of colitis. As compared to DSS, HCS was unaltered and DAI increased. Other variables were not significantly altered. This is in contrast to previous trials with dietary $n-3$ PUFA administration in experimental colitis, which has generally shown beneficial effects ${ }^{(27-29)}$. The fatty acid analysis of the diets confirmed large relative amounts of EPA and DHA in the two FO diets, with an $n-3: n-6$ ratio in the feed of 2.9 and $3 \cdot 1$.
The relatively anti-inflammatory eicosanoid $\mathrm{PGE}_{3}$ was increased, which is expected after dietary intake of EPA. In our study however, vitamin $\mathrm{E}$ or other anti-oxidants were not added to the diets containing FO. This could result in increased lipid peroxidation and oxidative stress in the colonic mucosa, which could counteract an anti-inflammatory effect $^{(30)}$. However, the levels of oxidation markers in our study were not increased, but rather tended to decrease, after the FO diet.

When combining FO and FP diets we observed that the $\mathrm{PGE}_{3}$ levels increased as compared to the FO diet. The reason for this is unclear, as adding the FP diet did not provide high amounts of EPA. FP, when combined with the FO diet, could possibly influence the enzymatic activity of cox2, thus leading to elevated $\mathrm{PGE}_{3}$ synthesis. We observed that the mRNA levels of ptgs $2 / \operatorname{cox} 2$ as well as ptges were elevated in the combined FO + FP group $v$. FO, but not reaching significance.

Nos2 expression was elevated after DSS $v$. controls, and was significantly increased after the FO + FP diet $v$. DSS alone. $\mathrm{NO}$ is a result of inducible NO synthase expression, and seems to have a dual role. It may provide either antiinflammatory or toxic effects ${ }^{(31)}$. Thus, the higher Nos2 levels after the $\mathrm{FO}+\mathrm{FP}$ diet may be a protective response, supported by the $\mathrm{PGE}_{3}$ and $\mathrm{PGD}_{2}$ increase, or it may be detrimental, as suggested by a tendency for elevated HCS and cytokine levels.

This study has its limitations: antioxidant added to the FO diets might have influenced the results. An increased number of rats in each group would have increased the statistical power of the analyses. Statistical analysis regarding feed and DSS intake could not be performed. We induced a severe colitis, leading to death in two animals, by administering $5 \%$ DSS for 1 week, which could limit the detection of modest beneficial effects. The diets used might affect the healing time of the colitis after stopping DSS. The effects observed may be due to prophylactic as well as therapeutic potential of the diets, as the study design cannot differentiate between these aspects. The DSS model clearly has limitations as a substitute for the human disease, as a chemically induced injury model instead of an endogenously triggered inflammation.

In conclusion, dietary hydrolysed salmon peptides may offer protection against DSS colitis as compared with a FO diet, but do not improve inflammatory variables significantly when compared with DSS alone. A combined diet may affect eicosanoid metabolism by increasing $\mathrm{PGE}_{3}$ levels, although not attenuating other signs of colon inflammation. Hydrolysed salmon peptides should be further investigated as a dietary intervention against inflammation in animal models, as well as in patients with IBD.

\section{Acknowledgements}

Ingeborg Kvivik, Kari Espedal and Marita Hanasand are thanked for their efforts during the cytokine analyses and Torunn Eide for the analyses of PG. We thank Eline Milde, Kari Williams and Randi Sandvik for excellent technical assistance. Jan Terje Kvaløy is thanked for valuable statistical advice. 
Contributions: T. G., B. B., T. H. and R. K. B. developed the study concept and design. T. G. and B. B. conducted the animal study, analysed and interpreted the data. T. G. drafted the manuscript. D. C., M. P. O. and R. P. carried out the oxidative marker analyses. O. G. A. conducted the histological evaluation, R. O. worked with cytokine analyses and A. S. carried out the PG analyses. P. B. measured the diet fatty acids. T. G., T. H., R. O. and R. B. K. made critical revisions of the manuscript. All authors read and approved the final manuscript.

The authors have no conflict of interest.

This work was supported by the Board of Nutrition Programmes (University of Bergen, Western Norway Regional Health Authority and the Institute of Marine Research). D. C. holds a fellowship from the Spanish Ministry of Health (FI0800707). Work carried out at the Department of Experimental Medicine was supported by grants from the Spanish Ministry of Science and Innovation (BFU2009-11879/BFI), the Autonomous Government of Catalonia (2009SGR735), and the Spanish Ministry of Health (PI081843) to M. P. O. and R. P. This work was also supported by COST B-35 action.

\section{References}

1. Meier J \& Sturm A (2011) Current treatment of ulcerative colitis World J Gastroenterol 17, 3204-3212.

2. Camuesco D, Galvez J, Nieto A, et al. (2005) Dietary olive oil supplemented with fish oil, rich in EPA and DHA (n-3) polyunsaturated fatty acids, attenuates colonic inflammation in rats with DSS induced colitis. J Nutr 135, 687-694.

3. Barros KV, Xavier RA, Abreu GG, et al. (2010) Soybean and fish oil mixture increases IL-10, protects against DNA damage and decreases colonic inflammation in rats with dextran sulfate sodium (DSS) colitis. Lipids Health Dis 9, 68.

4. Stenson WF, Cort D, Rodgers J, et al. (1992) Dietary supplementation with fish oil in ulcerative colitis. Ann Intern Med 116, 609-614.

5. Hawthorne AB, Daneshmend TK, Hawkey CJ, et al. (1992) Treatment of ulcerative colitis with fish oil supplementation: a prospective 12 month randomised controlled trial. Gut 33, 922-928.

6. Almallah YZ, Richardson S, O'Hanrahan T, et al. (1998) Distal procto-colitis, natural cytotoxicity, and essential fatty acids. $A m I$ Gastroenterol 93, 804-809.

7. Calder PC (2006) n-3 Polyunsaturated fatty acids, inflammation, and inflammatory diseases. Am J Clin Nutr 83, 1505S-1519S.

8. Endres S, Ghorbani R, Kelley VE, et al. (1989) The effect of dietary supplementation with $\mathrm{n}-3$ polyunsaturated fatty acids on the synthesis of interleukin-1 and tumor necrosis factor by mononuclear cells. N Engl J Med 320, 265-271.

9. Marchbank T, Elia G \& Playford RJ (2009) Intestinal protective effect of a commercial fish protein hydrolysate preparation. Regul Pept 155, 105-109.

10. Marchbank T, Limdi JK, Mahmood A, et al. (2008) Clinical trial: protective effect of a commercial fish protein hydrolysate against indomethacin (NSAID)-induced small intestinal injury. Aliment Pharmacol Ther 28, 799-804.

11. Thomas PD, Nichols TW \& Angstadt A (2001) Dietary bioactive peptides in maintaining intestinal integrity and function. $A m J$ Gastroenterol 96, S311.
12. Grimstad T, Berge RK, Bohov P, et al. (2011) Salmon diet in patients with active ulcerative colitis reduced the simple clinical colitis activity index and increased the anti-inflammatory fatty acid index - a pilot study. Scand J Clin Lab Invest 71, 68-73.

13. Rezaie A, Parker RD \& Abdollahi M (2007) Oxidative stress and pathogenesis of inflammatory bowel disease: an epiphenomenon or the cause? Dig Dis Sci 52, 2015-2021.

14. Je J-Y, Park P-J \& Kim S-K (2005) Antioxidant activity of a peptide isolated from Alaska pollack (Theragra chalcogramma) frame protein hydrolysate. Food Res Int 38, 45-50.

15. Parra D, Bandarra NM, Kiely M, et al. (2007) Impact of fish intake on oxidative stress when included into a moderate energy-restricted program to treat obesity. Eur J Nutr 46, 460-467.

16. Stucchi AF, Shofer S, Leeman S, et al. (2000) NK-1 antagonist reduces colonic inflammation and oxidative stress in dextran sulfate-induced colitis in rats. Am J Physiol Gastrointest Liver Physiol 279, G1298-G1306.

17. Grimstad T, Bjorndal B, Cacabelos D, et al. (2012) Dietary supplementation of krill oil attenuates inflammation and oxidative stress in experimental ulcerative colitis in rats. Scand J Gastroenterol 47, 49-58.

18. Carrier J, Aghdassi E, Platt I, et al. (2001) Effect of oral iron supplementation on oxidative stress and colonic inflammation in rats with induced colitis. Aliment Pharmacol Ther 15, 1989-1999.

19. Pamplona R, Portero-Otin M, Requena J, et al. (2002) Oxidative, glycoxidative and lipoxidative damage to rat heart mitochondrial proteins is lower after 4 months of caloric restriction than in agematched controls. Mech Ageing Dev 123, 1437-1446.

20. Andersen CL, Jensen JL \& Orntoft TF (2004) Normalization of real-time quantitative reverse transcription-PCR data: a modelbased variance estimation approach to identify genes suited for normalization, applied to bladder and colon cancer data sets. Cancer Res 64, 5245-5250.

21. de Medina FS, Daddaoua A, Requena P, et al. (2010) New insights into the immunological effects of food bioactive peptides in animal models of intestinal inflammation. Proc Nutr Soc 69, 454-462.

22. Coeffier M, Marion-Letellier R \& Dechelotte P (2009) Potential for amino acids supplementation during inflammatory bowel diseases. Inflamm Bowel Dis 16, 518-524.

23. Ryan JT, Ross RP, Bolton D, et al. (2011) Bioactive peptides from muscle sources: meat and fish. Nutrients 3, 765-791.

24. Hall LJ, Faivre E, Quinlan A, et al. (2010) Induction and activation of adaptive immune populations during acute and chronic phases of a murine model of experimental colitis. Dig Dis Sci 56, 79-89.

25. Ajuebor MN, Singh A \& Wallace JL (2000) Cyclooxygenase-2-derived prostaglandin $\mathrm{D}(2)$ is an early anti-inflammatory signal in experimental colitis. Am J Physiol Gastrointest Liver Physiol 279, G238-G244.

26. Vong L, Ferraz JG, Panaccione R, et al. (2010) A pro-resolution mediator, prostaglandin $\mathrm{D}(2)$, is specifically up-regulated in individuals in long-term remission from ulcerative colitis. Proc Natl Acad Sci U S A 107, 12023-12027.

27. Vilaseca J, Salas A, Guarner F, et al. (1990) Dietary fish oil reduces progression of chronic inflammatory lesions in a rat model of granulomatous colitis. Gut 31, 539-544.

28. Yuceyar H, Ozutemiz O, Huseyinov A, et al. (1999) Is administration of n-3 fatty acids by mucosal enema protective against trinitrobenzene-induced colitis in rats? Prostaglandins Leukot Essent Fatty Acids 61, 339-345.

29. Nieto N, Torres MI, Rios A, et al. (2002) Dietary polyunsaturated fatty acids improve histological and biochemical alterations in rats with experimental ulcerative colitis. J Nutr 132, 11-19.

30. Meydani SN (1996) Effect of (n-3) polyunsaturated fatty acids on cytokine production and their biologic function. Nutrition 12, S8-S14.

31. Cross RK \& Wilson KT (2003) Nitric oxide in inflammatory bowel disease. Inflamm Bowel Dis 9, 179-189. 